\title{
BER Analysis of Arbitrary QAM for MRC Diversity With Imperfect Channel Estimation in Generalized Ricean Fading Channels
}

\author{
Laleh Najafizadeh, Student Member, IEEE, and Chintha Tellambura, Senior Member, IEEE
}

\begin{abstract}
Imperfect channel estimation (ICE) can severely degrade the bit error rate (BER) of digital modulations with maximum ratio combining (MRC) diversity reception. The resulting performance analysis problem in its most general setting has not been addressed before. In this paper, the effect of ICE on the BER of an arbitrary square/rectangular Gray-coded quadratic amplitude modulation (QAM) in generalized Ricean fading channels when MRC reception is employed is analyzed. A general expression for the bit error probability of an arbitrary square/rectangular QAM scheme is first derived. This general formula requires a number of conditional probabilities, which is derived in closed form for independent and nonidentically distributed (i.n.d.) Rayleigh-fading channels with MRC and ICE. An efficient numerical method is also presented to compute the conditional probabilities for i.n.d. and correlated Ricean fading. In addition, extensive Monte Carlo simulations that agree excellently with the analytical results are presented.
\end{abstract}

Index Terms-Channel estimation, maximum ratio combining (MRC), quadratic amplitude modulation (QAM), Ricean fading.

\section{INTRODUCTION}

Q UADRATURE amplitude modulation (QAM), a highly spectrally efficient modulation scheme, is widely used in wireless-communication systems [1]. The bit error rate (BER) performance of $M$-ary QAM has been investigated by several authors. The exact BER for 16-QAM and 64-QAM in an additive white Gaussian noise (AWGN) channel is derived in [2]. Approximate expressions for the BER of an arbitrary $M$-ary square QAM using signal space concepts and recursive algorithms are given in [3] and [4], respectively. Cho and Yoon [5] derive a general expression for the BER of an arbitrary square/rectangular QAM in AWGN. In all these papers, the analysis assumes a perfectly known channel at the receiver.

For imperfect channel estimation (ICE), less work is available in the literature. Tang et al. [6] derive an approximate expression for the BER of 16-QAM and 64-QAM with ICE over Rayleigh-fading channels. In [7], a probability density

Manuscript received February 10, 2005; revised August 12, 2005 and October 25, 2005. This paper was presented in part at the 2005 IEEE International Conference on Communications, South Korea, May 2005. The review of this paper was coordinated by Prof. C. Tepedelenlioglu.

L. Najafizadeh was with the Department of Electrical and Computer Engineering, University of Alberta, Edmonton, AB T6G 2V4, Canada. She is now with the School of Electrical and Computer Engineering, Georgia Institute of Technology, Atlanta, GA 30332 USA (e-mail: laleh@ece.gatech.edu).

C. Tellambura is with the Department of Electrical and Computer Engineering, University of Alberta, Edmonton, AB T6G 2V4, Canada (e-mail: chintha@ece.ualberta.ca).

Digital Object Identifier 10.1109/TVT.2006.874573 function (pdf) is introduced to determine the BER of maximum ratio combining (MRC) QAM with channel estimation error. However, the closed-form integrals are obtained only for a 16-QAM constellation with one and two diversity branches for Rayleigh fading and for one branch for Ricean fading. Cao and Beaulieu [8], [9] provide exact BER expressions for a 16-QAM MRC receiver in Rayleigh and Ricean fading channels with any number of diversity branches. Their results are only valid for independent and identically distributed (i.i.d.) fading channels. Furthermore, the results in [6]-[9] are presented only for a specific square QAM constellation.

Previously, the performance of $M$-ary phase shift keying (M-PSK) with MRC and ICE in generalized Ricean fading channels [10] and with general selection combining (GSC) and equal gain combining (EGC) receivers with Gaussian weighting errors for several types of i.i.d. fading channels [11] was investigated. In [12], we analyzed the BER performance of square/rectangular QAM with MRC and ICE in Rayleighfading channels. The results in [12] are extended here for the computation of the BER of an arbitrary square/rectangular QAM in generalized Ricean fading channels.

In this paper, we present a general expression for the BER of an arbitrary square/rectangular QAM. First, by analyzing the BER performance of 16 QAM, we observe a regular pattern for the bit error probability of the $k$ th in-phase bit. From this pattern, we develop a general expression for the BER performance of an arbitrary $M$-ary square QAM receiver. This general expression is then extended to an arbitrary $I \times J$-ary rectangular QAM.

The general expressions obtained in this paper require a number of conditional probabilities, which depend on the channel statistics and the decision variable. For MRC with ICE in i.i.d. fading channels, our several conditional probabilities reduce to the formula provided in [13, Appendix B]. A special case (16 QAM) is considered in [9]. For MRC with ICE in independent and nonidentically distributed (i.n.d.) Rayleighfading channels, no BER expression is available in literature. Therefore, we derive a closed-form expression for the BER of an arbitrary square/rectangular QAM constellation with MRC and ICE in i.n.d. Rayleigh-fading channels. We also present an efficient numerical technique for the computation of the BER of any square/rectangular QAM in i.n.d. and correlated Ricean fading channels. This technique is based on a classic result due to Imhof [14], which provides a numerical algorithm for the distribution of a quadratic form in normal variables. 
This paper is organized as follows. The system and QAM signaling models and assumptions are described in Section II. In Section III, we derive a regular pattern for the BER of an arbitrary square QAM in terms of conditional error probabilities, and, in Section IV, the pattern is extended to an arbitrary rectangular QAM. A closed-form error-rate expression for an arbitrary square/rectangular QAM in i.n.d. Rayleigh-fading channels as well as a numerical technique for computing its BER in i.n.d. and correlated Ricean fading channels, all cases with MRC and ICE, are presented in Section V. In Section VI, numerical examples are given, and the validity of our analytical results is assessed using the Monte Carlo simulations. The conclusion is given in Section VII.

\section{System AND Signal Models}

\section{A. System Model}

The system model used here is identical to that considered in [7]. MRC is used at the receiver to combat the effects of fading. The received signal sample at each receiver antenna is given by

$$
y_{m}=h_{m} x+n_{m}, \quad m=1, \ldots, L
$$

where $x=x_{I}+j x_{Q}, j=\sqrt{-1}, x_{I} \in\{ \pm d, \pm 3 d, \ldots, \pm(I-$ 1)d\}, $x_{Q} \in\{ \pm d, \pm 3 d, \ldots, \pm(J-1) d\}$, is the data symbol chosen from an $I \times J$ rectangular QAM constellation, $L$ is the number of diversity branches, and $h_{m}$ and $n_{m}$ denote the channel attenuation and AWGN of the $m$ th branch, respectively. The channel $h_{m}$ can be estimated by transmitting pilot symbols [15], [16]. At the receiver, the channel estimator outputs

$$
g_{m}=\alpha h_{m}+s_{m}, \quad m=1, \ldots, L
$$

where $\alpha$ is a complex number representing the bias in the estimate, and $s_{m}$ is a circularly complex Gaussian random variable (RV) with mean zero and variance $\sigma_{S}^{2}$ representing the random noise in the estimate; note that $s_{m}$ and $h_{m}$ are uncorrelated. We assume that all branch noise variances are equal, i.e., $E\left|n_{m}\right|^{2}=$ constant for $m=1, \ldots, L$.

The MRC decision variable at the receiver is given in [7]

$$
z_{d}=\frac{\sum_{m=1}^{L} y_{m} g_{m}^{*}}{\sum_{m=1}^{L}\left|g_{m}\right|^{2}} .
$$

The superscript * represents the conjugate operation. Since we only consider two-dimensional (2-D) modulation schemes where the in-phase $(I)$ and quadrature $(Q)$ streams can be demodulated separately, we need the real and imaginary components of $z_{d}$

$$
z_{d_{I}}=\operatorname{Re}\left(z_{d}\right)=\frac{\sum_{m=1}^{L}\left(y_{m} g_{m}^{*}+y_{m}^{*} g_{m}\right)}{2 \sum_{m=1}^{L}\left|g_{m}\right|^{2}}
$$

and

$$
z_{d_{Q}}=\operatorname{Im}\left(z_{d}\right)=\frac{\sum_{m=1}^{L}\left(y_{m} g_{m}^{*}-y_{m}^{*} g_{m}\right)}{2 j \sum_{m=1}^{L}\left|g_{m}\right|^{2}} .
$$

Define $\bar{W}=\left(g_{1}, y_{1}, g_{2}, y_{2}, \ldots, g_{L}, y_{L}\right)^{t}$ and $\bar{\mu}=E[\bar{W}]=$ $\left[\mu_{g_{1}}, \mu_{y_{1}}, \ldots, \mu_{g_{L}}, \mu_{y_{L}}\right]^{t}$, where $t$ represents the transpose operation, and

$$
\begin{aligned}
& \mu_{g_{m}}=\alpha E\left(h_{m}\right) \\
& \mu_{y_{m}}=x E\left(h_{m}\right) .
\end{aligned}
$$

We find the covariance matrix of $\bar{W}$ as follows:

$$
\begin{aligned}
& C_{W}=E\left[(\bar{W}-\bar{\mu})(\bar{W}-\bar{\mu})^{\dagger}\right] \\
& =\left(\begin{array}{ccccccc}
\sigma_{g_{1} g_{1}}^{2} & \sigma_{g_{1} y_{1}}^{2} & \sigma_{g_{1} g_{2}}^{2} & \sigma_{g_{1} y_{2}}^{2} & \cdots & \sigma_{g_{1} g_{L}}^{2} & \sigma_{g_{1} y_{L}}^{2} \\
\sigma_{y_{1} g_{1}}^{2} & \sigma_{y_{1} y_{1}}^{2} & \sigma_{y_{1} g_{2}}^{2} & \sigma_{y_{1} y_{2}}^{2} & \cdots & \sigma_{y_{1} g_{L}}^{2} & \sigma_{y_{1} y_{L}}^{2} \\
\vdots & \vdots & \vdots & \vdots & & \vdots & \vdots \\
\sigma_{g_{L} g_{1}}^{2} & \sigma_{g_{L} g_{1}}^{2} & \sigma_{g_{L} g_{2}}^{2} & \sigma_{g_{L} y_{2}}^{2} & \cdots & \sigma_{g_{L} g_{L}}^{2} & \sigma_{g_{L} y_{L}}^{2} \\
\sigma_{y_{L} g_{1}}^{2} & \sigma_{y_{L} y_{1}}^{2} & \sigma_{y_{L} g_{2}}^{2} & \sigma_{y_{L} y_{2}}^{2} & \cdots & \sigma_{y_{L} g_{L}}^{2} & \sigma_{y_{L} y_{L}}^{2}
\end{array}\right)
\end{aligned}
$$

where the superscript ${ }^{\dagger}$ represents the conjugate transpose operation. The quantities $\sigma_{g_{m} g_{n}}^{2}, \sigma_{y_{m} y_{n}}^{2}$, and $\sigma_{g_{m} y_{n}}^{2}$ are defined as

$$
\begin{aligned}
\sigma_{g_{m} g_{n}}^{2} & =E\left(\left(g_{m}-\mu_{g_{m}}\right)\left(g_{n}-\mu_{g_{n}}\right)^{*}\right) \\
& = \begin{cases}|\alpha|^{2} \sigma_{h_{m} h_{m}}^{2}+E\left(\left|s_{m}\right|^{2}\right) & (m=n) \\
|\alpha|^{2} \sigma_{h_{m} h_{n}}^{2} & (m \neq n)\end{cases} \\
\sigma_{y_{m} y_{n}}^{2} & =E\left(\left(y_{m}-\mu_{y_{m}}\right)\left(y_{n}-\mu_{y_{n}}\right)^{*}\right) \\
& = \begin{cases}|x|^{2} \sigma_{h_{m} h_{m}}^{2}+E\left(\left|n_{m}\right|^{2}\right) & (m=n) \\
|x|^{2} \sigma_{h_{m} h_{n}}^{2} & (m \neq n)\end{cases} \\
\sigma_{g_{m} y_{n}}^{2} & =E\left(\left(g_{m}-\mu_{g_{m}}\right)\left(y_{n}-\mu_{y_{n}}\right)^{*}\right)=\alpha x^{*} \sigma_{h_{m} h_{n}}^{2}
\end{aligned}
$$

where $\sigma_{h_{m} h_{n}}^{2}=E\left(h_{m} h_{n}^{*}\right)-\mu_{h_{m}} \mu_{h_{n}}^{*}$. When the branches $m$ and $n$ are uncorrelated, $\sigma_{h_{m} h_{n}}^{2}=0$. Also, note that $\sigma_{g_{m} y_{n}}=\sigma_{y_{n} g_{m}}^{*}$.

\section{B. QAM Signaling}

We consider $I \times J$ rectangular QAM consisting of two independent one-dimensional pulse amplitude modulation (PAM) signals. In the special case when $I=J$, the constellation represents an $M$-ary square QAM, where $M=I \times I$. We also assume that all the symbols are equally likely to be transmitted.

In a typical QAM modulator, the data stream is divided into the $I$ and $Q$ bit streams, each encoded onto a separate axis using identical Gray coding mapping blocks [1]. The in-phase and quadrature components are thus selected independently over the set of $\{ \pm d, \pm 3 d, \ldots, \pm(I-1) d\}$ and $\{ \pm d, \pm 3 d, \ldots, \pm(J-$ $1) d\}$, respectively. Note that $2 d$ is the Euclidean distance between two adjacent signal points. Denoting $E_{b}$ as the bit energy, $d$ can be written in terms of $E_{b}, I$ and $J$ as [5]

$$
d=\sqrt{\frac{3 E_{b} \log _{2}(I \times J)}{I^{2}+J^{2}-2}} .
$$


For the case of $M$-ary square QAM, (8) becomes

$$
d=\sqrt{\frac{3 E_{b} \log _{2} M}{2(M-1)}} .
$$

At the receiver, the complex symbols are divided into $I$ and $Q$ components, and each component is demodulated independently against their respective decision boundaries.

We next derive a general BER expression for $M$-ary square and $I \times J$ rectangular QAM in terms of conditional probabilities.

\section{GENERAL BER EXPRESSION FOR $M$-ARY SQUARE QAM}

A general expression for the BER of an arbitrary square/ rectangular QAM for AWGN channels is derived in [5]. However, this expression depends on the complementary error function and is only valid for AWGN channels. Here, we derive a general expression for the BER of $M$-ary QAM, which is valid not only in AWGN but also in any fading channel with/without diversity and with perfect/ICE.

Due to symmetry, the average BER of an $M$-ary square QAM is equal to the BER of either the $I$ or the $Q$ components. We assume that in the QAM constellation, the most significant bit is the in-phase bit. Then, the BER of an $M$-ary square QAM is given in [5, eq. (16)]

$$
P_{b}=\frac{1}{\log _{2} \sqrt{M}} \sum_{n=0}^{\log _{2} \sqrt{M}-1} P_{I}\left(\log _{2} M-2 n\right)
$$

where $P_{I}\left(\log _{2} M-2 n\right)$ denotes the bit error probability of the $\left(\log _{2} M-2 n\right)$ th bit in the in-phase components. Therefore, in order to obtain the average BER of an $M$-ary square QAM, one needs to determine $P_{I}\left(\log _{2} M-2 n\right)$. In this section, we first determine $P_{I}\left(\log _{2} M-2 n\right)$ for the two in-phase bits of a 16-QAM constellation. Using this example, we then obtain a general expression for the bit error probability of the $\left(\log _{2} M-\right.$ $2 n)$ th in-phase bit of an arbitrary $M$-ary square QAM. Then, using (10), the BER of an $M$-ary square QAM is determined.

\section{A. BER of $16 Q A M$}

Fig. 1 shows Gray-coded bit mapping and the decision boundaries for square 16 QAM. The fourth ${ }^{1}$ and the second bits are the in-phase bits while the third and the first bits are the quadrature bits. Due to symmetry, we only consider the bit error probability of the in-phase bits. Note that with ICE, the decision boundaries shown in Fig. 1 may no longer be optimal in the sense of minimizing the overall error rate. In [17] and [18], an optimal metric that takes ICE into account has been derived, the use of which is equivalent to modifying the decision boundaries

\footnotetext{
${ }^{1}$ We refer to the first bit as the least significant bit and to the $\left(\log _{2} M\right)$ th bit as the most significant bit.
}

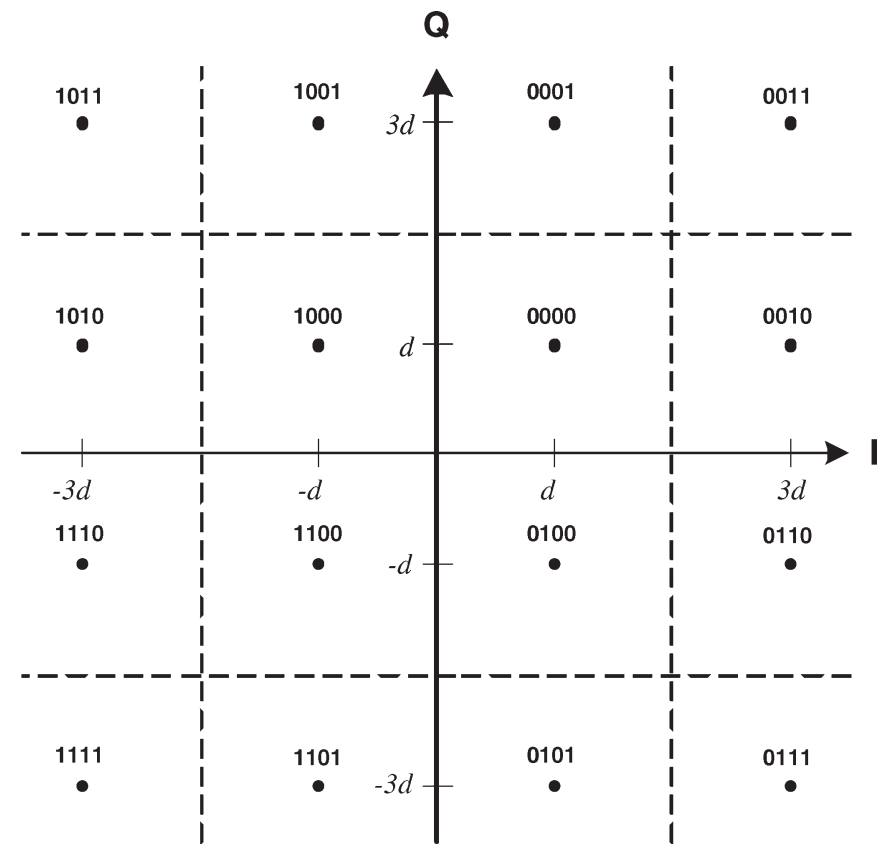

Fig. 1. Square 16-QAM constellation with gray encoding.

in Fig. 1. Our simulations reveal that the use of this metric has a negligible effect on the error performance of uncoded systems especially when the estimation noise variance is small compared with the channel noise variance. Consequently, we do not use the optimal metric derived in [17] and [18].

The Q-axis is the decision boundary for the fourth bit. Because of the symmetry between the right and left half sides of the plane, the probability that the fourth bit is in error is [2]

$$
\begin{aligned}
P_{I}(4)= & \frac{1}{2}\left[\operatorname{Pr}\left(z_{d_{I}}<\left.0\right|_{\operatorname{Re}(x)=d}\right)+\operatorname{Pr}\left(z_{d_{I}}<\left.0\right|_{\operatorname{Re}(x)=3 d}\right)\right] \\
= & \frac{1}{4}\left[\operatorname{Pr}\left(z_{d_{I}}<\left.0\right|_{x=d+j d}\right)+\operatorname{Pr}\left(z_{d_{I}}<\left.0\right|_{x=d+j 3 d}\right)\right. \\
& \left.\quad+\operatorname{Pr}\left(z_{d_{I}}<\left.0\right|_{x=3 d+j d}\right)+\operatorname{Pr}\left(z_{d_{I}}<\left.0\right|_{x=3 d+j 3 d}\right)\right] .
\end{aligned}
$$

For the second bit, the decision boundaries are $2 d$ and $-2 d$. If the second bit is an " 0 ," an error occurs when the real part of the decision variable $z_{d}$ is out of the region $(-2 d, 2 d)$, and if the second bit is " 1 ," an error occurs if the real part of $z_{d}$ falls in $(-2 d, 2 d)$. Therefore, the error probability of the second bit is obtained as [2]

$$
\begin{aligned}
& P_{I}(2) \\
& \begin{aligned}
=\frac{1}{2} & {\left[\operatorname{Pr}\left(\left|z_{d_{I}}\right|>\left.2 d\right|_{\operatorname{Re}(x)=d}\right)+\operatorname{Pr}\left(\left|z_{d_{I}}\right|<\left.2 d\right|_{\operatorname{Re}(x)=3 d}\right)\right] } \\
=\frac{1}{4} & {\left[1-\operatorname{Pr}\left(z_{d_{I}}<\left.2 d\right|_{x=d+j d}\right)-\operatorname{Pr}\left(z_{d_{I}}<\left.2 d\right|_{x=d+j 3 d}\right)\right.} \\
& +1+\operatorname{Pr}\left(z_{d_{I}}<-\left.2 d\right|_{x=d+j d}\right)+\operatorname{Pr}\left(z_{d_{I}}<-\left.2 d\right|_{x=d+j 3 d}\right) \\
& +\operatorname{Pr}\left(z_{d_{I}}<\left.2 d\right|_{x=3 d+j d}\right)+\operatorname{Pr}\left(z_{d_{I}}<\left.2 d\right|_{x=3 d+j 3 d}\right) \\
& \left.\quad-\operatorname{Pr}\left(z_{d_{I}}<-\left.2 d\right|_{x=3 d+j d}\right)-\operatorname{Pr}\left(z_{d_{I}}<-\left.2 d\right|_{x=3 d+j 3 d}\right)\right] .
\end{aligned}
\end{aligned}
$$

Substituting (11) and (12) into (10), the BER for 16 QAM is obtained. 


\section{B. General BER Expression for an $M$-ary Square QAM}

From (11) and (12), a regular pattern can be found for the bit error probability of the $\left(\log _{2} M-2 n, n \in\{0,1, \ldots\right.$, $\left.\left.\log _{2} \sqrt{M}-1\right\}\right)$ th bit as

$$
\begin{aligned}
& P_{I}\left(\log _{2} M-2 n\right) \\
& = \begin{cases}\frac{4}{M}\left[\sum_{\substack{k=1 \\
k \text { odd }}}^{\sqrt{M}-1} A_{k, 0}\right] & (n=0) \\
\frac{4}{M}\left[\frac{M}{8}+\sum_{i=0}^{2^{n-1}} \sum_{\substack{k=F_{1}(i, n) \\
k \text { odd }}}^{F_{2}(i, n)}(-1)^{i} A_{k, n}\right] & (n \neq 0)\end{cases}
\end{aligned}
$$

where $F_{1}(i, n)=\max \left[\left(\sqrt{M} / 2^{n}\right)(2 i-1)+1,1\right], F_{2}(i, n)=$ $\min \left[\left(\sqrt{M} / 2^{n}\right)(2 i+1)-1, \sqrt{M}-1\right]$, and

$A_{k, n}$

$$
=\left\{\begin{array}{cc}
\sum_{\substack{l=1 \\
l \text { odd }}}^{\sqrt{M}-1}\left[\operatorname{Pr}\left(z_{d I}<\left.0\right|_{x=k d+j l d}\right)\right] & (n=0) \\
\sum_{\substack{M=1 \\
l \text { odd }}}^{\sqrt{M}} \sum_{i=0}^{n-1}(-1)^{i}\left[\operatorname{Pr}\left(z_{d I}<-\left.G_{i, n}\right|_{x=k d+j l d}\right)\right. & \\
\left.-\operatorname{Pr}\left(z_{d I}<\left.G_{i, n}\right|_{x=k d+j l d}\right)\right] & (n \neq 0)
\end{array}\right.
$$

where $G_{i, n}=\left(\sqrt{M}(2 i+1) / 2^{n}\right) d$.

If the conditional probabilities are known, then the above expressions for $P_{I}\left(\log _{2} M-2 n\right)$ can be used in (10) to obtain the BER of an $M$-ary square QAM.

To compare our result with the expression derived in [5], we consider an AWGN channel without fading, for which the decision variable $z_{d}$ reduces to

$$
z_{d}=x+n_{0}
$$

where $n_{0}$ is a zero-mean AWGN with a two-sided power spectrum density $N_{0} / 2$. The bit error probability of the most significant bit $(n=0)$, for example, is obtained from (13) as

$$
P_{I}\left(\log _{2} M\right)=\frac{1}{\sqrt{M}}\left[\sum_{\substack{k=1 \\ k \text { odd }}}^{\sqrt{M}-1} \operatorname{erfc}\left(\frac{k d}{\sqrt{N_{0}}}\right)\right]
$$

which is equal to [5, eq. (15)]. ${ }^{2}$

\section{General BER EXPRESSION FOR $I \times J$ RECTANGULAR QAM}

The previous section derives a general expression for BER of $M$-ary square QAM. In this section, we extend this analysis to an arbitrary $I \times J$ rectangular QAM.

\footnotetext{
${ }^{2}$ Note that in [5] the most significant bit is referred to as first bit.
}

If we assume that the most significant bit is the in-phase bit, then the BER of an $I \times J$ rectangular QAM is given by [5, eq. (22)]

$$
\begin{array}{r}
P_{b}=\frac{1}{\log _{2}(I \times J)} \times\left[\sum_{n=0}^{\log _{2} I-1} P_{I}\left(\log _{2}(I \times J)-2 n\right)\right. \\
\left.+\sum_{n=0}^{\log _{2} J-1} P_{Q}\left(\log _{2}(I \times J)-(2 n+1)\right)\right]
\end{array}
$$

where $P_{I}\left(\log _{2}(I \times J)-2 n\right)$ and $P_{Q}\left(\log _{2}(I \times J)-(2 n+\right.$ 1)) denote the bit error probabilities of the $\left(\log _{2}(I \times J)-\right.$ $2 n)$ th in-phase bit and $\left(\log _{2}(I \times J)-(2 n+1)\right)$ th quadrature bit, respectively. The general expression for the BER of the inphase bits is given in Section III. Note that for the case of $I \times J$ rectangular QAM, $M$ and $\sqrt{M}$ in (13) should be replaced by $I \times J$ and $I$, respectively.

For the quadrature bits, the imaginary part of the decision variable should be considered. The regular pattern that can be found for the bit error probability of the $\left(\log _{2} I \times J-\right.$ $(2 n+1))$ th bit is

$$
\begin{aligned}
& P_{Q}\left(\log _{2}(I \times J)-(2 n+1)\right) \\
& = \begin{cases}\frac{4}{I \times J}\left[\begin{array}{c}
J-1 \\
\left.\sum_{k=1}^{J} B_{k, 0}\right]
\end{array}\right. & (n=0) \\
\frac{4}{I \times J}\left[\frac{I \times J}{8}+\sum_{i=0}^{2^{n-1}} \sum_{\substack{k=E_{1}(i, n) \\
k \text { odd }}}^{E_{2}(i, n)}(-1)^{i} B_{k, n}\right] & (n \neq 0)\end{cases}
\end{aligned}
$$

where $\quad E_{1}(i, n)=\max \left[\left(J / 2^{n}\right)(2 i-1)+1,1\right], \quad E_{2}(i, n)=$ $\min \left[\left(J / 2^{n}\right)(2 i+1)-1, J-1\right]$, and

$$
\begin{aligned}
& B_{k, n} \\
& =\left\{\begin{array}{cc}
\sum_{\substack{l=1 \\
l=1}}^{J-1}\left[\operatorname{Pr}\left(z_{d Q}<\left.0\right|_{x=l d+j k d}\right)\right] & (n=0) \\
\sum_{\substack{l=1 \\
l \text { odd }}}^{J-1} \sum_{i=0}^{n-1}(-1)^{i}\left[\operatorname{Pr}\left(z_{d Q}<-\left.V_{i, n}\right|_{x=l d+j k d}\right)\right. & \\
\left.-\operatorname{Pr}\left(z_{d Q}<\left.V_{i, n}\right|_{x=l d+j k d}\right)\right] & (n \neq 0)
\end{array}\right.
\end{aligned}
$$

where $V_{i, l}=\left(J(2 i+1) / 2^{l}\right) d$.

Once $P_{I}\left(\log _{2}(I \times J)-2 n\right)$ and $P_{Q}\left(\log _{2}(I \times J)-(2 n+\right.$ 1)) are defined from (13) and (17), the BER of an $I \times J$ rectangular QAM follows from (16).

The above analysis shows that the BER of an arbitrary square/rectangular QAM consists of the summation of the probability terms in the form of $\operatorname{Pr}\left(z_{d_{I}}<\left.T\right|_{x=a d+j b d}\right)$ and $\operatorname{Pr}\left(z_{d_{Q}}<\left.t\right|_{x=a d+j b d}\right)$, where $a \in\{1,3, \ldots,(I-1)\}, b \in$ $\{1,3, \ldots,(J-1)\}$, and $T$ depends on $d$. We next determine the expression for these probabilities when MRC with ICE is used at the receiver. 


\section{EXACT BER EXPRESSION FOR AN ARBITRARY SQUARE/RECTANGULAR QAM IN GENERALIZED RICEAN FADING CHANNELS}

The previous sections show that the BER of an arbitrary square/rectangular QAM can be written as a sum of probabilities in the form of $\operatorname{Pr}\left(z_{d_{I}}<\left.T\right|_{x=a d+j b d}\right)$ and $\operatorname{Pr}\left(z_{d_{Q}}<\right.$ $\left.\left.T\right|_{x=a d+j b d}\right)$. When MRC with ICE is employed, the decision variable $z_{d}$ is defined as (3).

Using (4a), the $\operatorname{Pr}\left(z_{d_{I}}<\left.T\right|_{x=a d+j b d}\right)$ can be rewritten as

$$
\begin{aligned}
& \operatorname{Pr}\left(z_{d I}<\left.T\right|_{x=a d+j b d}\right) \\
& \quad=\operatorname{Pr}\left(\frac{\sum_{m=1}^{L}\left(y_{m} g_{m}^{*}+y_{m}^{*} g_{m}\right)}{2 \sum_{m=1}^{L}\left|g_{m}\right|^{2}}<\left.T\right|_{x=a d+j b d}\right) \\
& \quad=\operatorname{Pr}\left(\sum_{m=1}^{L}\left(-2 T\left|g_{m}\right|^{2}+y_{m} g_{m}^{*}+y_{m}^{*} g_{m}\right)<\left.0\right|_{x=a d+j b d}\right) \\
& =\operatorname{Pr}\left(D_{I}<\left.0\right|_{x=a d+j b d}\right)
\end{aligned}
$$

where

$$
\begin{aligned}
D_{I} & =\sum_{m=1}^{L}\left(-2 T\left|g_{m}\right|^{2}+y_{m} g_{m}^{*}+y_{m}^{*} g_{m}\right) \\
& =\bar{W}^{\dagger} H_{I} \bar{W} \\
\bar{W} & =\left[g_{1}, y_{1}, g_{2}, y_{2}, \ldots, g_{L}, y_{L}\right]^{t}
\end{aligned}
$$

and

$$
H_{I}=\left(\begin{array}{ccccccc}
-2 T & 1 & 0 & 0 & \ldots & 0 & 0 \\
1 & 0 & 0 & 0 & \ldots & 0 & 0 \\
0 & 0 & -2 T & 1 & \ldots & 0 & 0 \\
0 & 0 & 1 & 0 & \ldots & 0 & 0 \\
\vdots & \vdots & \vdots & \vdots & & \vdots & \vdots \\
0 & 0 & 0 & 0 & \ldots & -2 T & 1 \\
0 & 0 & 0 & 0 & \ldots & 1 & 0
\end{array}\right) .
$$

Similarly, using (4b), $\operatorname{Pr}\left(z_{d_{Q}}<\left.T\right|_{x=a d+j b d}\right)$ can be expressed as

$$
\begin{aligned}
\operatorname{Pr} & \left(z_{d Q}<\left.T\right|_{x=a d+j b d}\right) \\
& =\operatorname{Pr}\left(\frac{\sum_{m=1}^{L}\left(y_{m} g_{m}^{*}-y_{m}^{*} g_{m}\right)}{2 j \sum_{m=1}^{L}\left|g_{m}\right|^{2}}<\left.T\right|_{x=a d+j b d}\right) \\
& =\operatorname{Pr}\left(\sum_{m=1}^{L}\left(-2 T\left|g_{m}\right|^{2}-j y_{m} g_{m}^{*}+j y_{m}^{*} g_{m}\right)<\left.0\right|_{x=a d+j b d}\right) \\
& =\operatorname{Pr}\left(D_{Q}<\left.0\right|_{x=a d+j b d}\right)
\end{aligned}
$$

where

$$
D_{Q}=\sum_{m=1}^{L}\left(-2 T\left|g_{m}\right|^{2}-j y_{m} g_{m}^{*}+j y_{m}^{*} g_{m}\right)=\bar{W}^{\dagger} H_{Q} \bar{W}
$$

and

$$
H_{Q}=\left(\begin{array}{ccccccc}
-2 T & \frac{1}{j} & 0 & 0 & \ldots & 0 & 0 \\
-\frac{1}{j} & 0 & 0 & 0 & \ldots & 0 & 0 \\
0 & 0 & -2 T & \frac{1}{j} & \ldots & 0 & 0 \\
0 & 0 & -\frac{1}{j} & 0 & \ldots & 0 & 0 \\
\vdots & \vdots & \vdots & \vdots & & \vdots & \vdots \\
0 & 0 & 0 & 0 & \ldots & -2 T & \frac{1}{j} \\
0 & 0 & 0 & 0 & \ldots & -\frac{1}{j} & 0
\end{array}\right) .
$$

Note that $D_{I}$ and $D_{Q}$ are special cases of the general quadratic form. Following [19], we can immediately write the moment generating functions (MGF) of $D_{I}$ and $D_{Q}$ as follows ${ }^{3}$ :

$$
\varphi_{D_{I}}(s)=\frac{1}{\left|I-s C_{W} H_{I}\right|} \exp \left\{\bar{\mu}^{\dagger}\left(s^{-1} H_{I}^{-1}-C_{W}\right)^{-1} \bar{\mu}\right\}
$$

and

$$
\varphi_{D_{Q}}(s)=\frac{1}{\left|I-s C_{W} H_{Q}\right|} \exp \left\{\bar{\mu}^{\dagger}\left(s^{-1} H_{Q}^{-1}-C_{W}\right)^{-1} \bar{\mu}\right\}
$$

where $C_{W}$ is the covariance matrix defined in (6).

Equations (25a) and (25b) can also be written in another useful form. Since $C_{W}$ is the covariance matrix, we can obtain its square root matrix denoted by $C_{W}^{1 / 2}$ such that $C_{W}=C_{W}^{1 / 2} C_{W}^{1 / 2}$. Then, if we define $\bar{V}=C_{W}^{-1 / 2} \bar{W}$, where $C_{W}^{-1 / 2}$ is the inverse of $C_{W}^{1 / 2},(19)$ and (23) can be written as

$$
\begin{aligned}
D_{I} & =\bar{V}^{\dagger}\left(C_{W}^{1 / 2} H_{I} C_{W}^{1 / 2}\right) \bar{V} \\
D_{Q} & =\bar{V}^{\dagger}\left(C_{W}^{1 / 2} H_{Q} C_{W}^{1 / 2}\right) \bar{V} .
\end{aligned}
$$

Using an eigenvalue decomposition, we rewrite (26a) and (26b) as

$$
\begin{aligned}
D_{I} & =\bar{V}^{\dagger}\left(U_{I} \Lambda_{I} U_{I}^{\dagger}\right) \bar{V} \\
D_{Q} & =\bar{V}^{\dagger}\left(U_{Q} \Lambda_{Q} U_{Q}^{\dagger}\right) \bar{V}
\end{aligned}
$$

where $\Lambda_{I}=\operatorname{diag}\left(\lambda_{a_{1}}, \lambda_{a_{2}}, \ldots, \lambda_{a_{2 L}}\right), \Lambda_{Q}=\operatorname{diag}\left(\lambda_{b_{1}}, \lambda_{b_{2}}\right.$, $\left.\ldots, \lambda_{b_{2 L}}\right)$, and $\lambda_{a_{i}}$ and $\lambda_{b_{i}}$ are the eigenvalues of $C_{W}^{1 / 2} H_{I} C_{W}^{1 / 2}$ and $C_{W}^{1 / 2} H_{Q} C_{W}^{1 / 2}$, respectively.

Let $\bar{X}_{I}=U_{I}^{\dagger} C_{W}^{-1 / 2} \bar{\mu}$ and $\bar{X}_{Q}=U_{Q}^{\dagger} C_{W}^{-1 / 2} \bar{\mu}$. $D_{I}$ and $D_{Q}$ in (27a) and (27b) can now be written as a weighted sum of

${ }^{3}$ Note that we have defined the covariance matrix of a vector $\bar{W}$ as $E\left(\bar{W} \bar{W}^{\dagger}\right)$ rather than $(1 / 2) E\left(\bar{W} \bar{W}^{\dagger}\right)$ as in [19]. 
noncentral chi-square RVs. Using [13], the MGFs of $D_{I}$ and $D_{Q}$ are expressed as

$$
\begin{aligned}
\varphi_{D_{I}}(s) & =\prod_{r=1}^{2 L}\left(\frac{1}{1-s \lambda_{a_{r}}}\right) \exp \left(\frac{s\left|x_{I_{r}}\right|^{2} \lambda_{a_{r}}}{1-s \lambda_{a_{r}}}\right) \\
\varphi_{D_{I}}(s) & =\prod_{r=1}^{2 L}\left(\frac{1}{1-s \lambda_{b_{r}}}\right) \exp \left(\frac{s\left|x_{Q_{r}}\right|^{2} \lambda_{b_{r}}}{1-s \lambda_{b_{r}}}\right) .
\end{aligned}
$$

When repeated eigenvalues exist, the MGF expressions (28a) and (28b) are modified as [20]

$$
\begin{gathered}
\varphi_{D_{I}}(s)=\prod_{r=1}^{N}\left(\frac{1}{1-s \lambda_{a_{r}}}\right)^{v_{i_{r}}} \exp \left(\frac{y_{I_{r}}^{2} \lambda_{a_{r}} s}{1-s \lambda_{a_{r}}}\right) \\
\varphi_{D_{Q}}(s)=\prod_{r=1}^{N}\left(\frac{1}{1-s \lambda_{b_{r}}}\right)^{v_{q_{r}}} \exp \left(\frac{y_{Q_{r}}^{2} \lambda_{b_{r}} s}{1-s \lambda_{b_{r}}}\right)
\end{gathered}
$$

where $N$ is the total number of distinct eigenvalues, $v_{i_{r}}$ and $v_{q_{r}}$ are the multiplicity of the eigenvalues $\lambda_{a_{r}}$ and $\lambda_{b_{r}}$, respectively, and

$$
\begin{gathered}
y_{I_{r}}^{2}=\sum_{k \in \kappa_{r}}\left|x_{I_{r}}\right|^{2} \\
y_{Q_{r}}^{2}=\sum_{k \in \kappa_{r}}\left|x_{Q_{r}}\right|^{2}
\end{gathered}
$$

and $\kappa_{r}$ denotes the set of $k$ indexes associated with the $r$ th distinct eigenvalue.

We now derive the BER expression for an arbitrary square/ rectangular QAM in the generalized fading channel.

\section{A. i.i.d. Fading Channels}

With the assumption of i.i.d. fading statistics across the diversity branches, $h_{m}$ and $g_{m}$ in (3) are i.i.d. Gaussian RVs. With MRC in i.i.d. Rayleigh or Ricean fading channels, the conditional probabilities in (13) and (17) are reduced to a closed-form expression given in [13, eq. (B-21)]. Our general expression then can be used for the derivation of the BER of any square/rectangular QAM scheme with MRC and ICE in i.i.d. Rayleigh/ Ricean fading channels. A special case of 16 QAM is considered in [9].

\section{B. i.n.d. Fading Channels}

The fading statistics are different across the diversity branches. This occurs when different signals may propagate via completely different paths before arriving at the receiver.

For the i.n.d. case, we derive a closed-form error-rate expression for Rayleigh fading and present a numerical technique for Ricean fading to compute the BER performance of an arbitrary square/rectangular QAM with MRC and ICE.
1) Rayleigh Fading: In a Rayleigh-fading channel, we have $\bar{\mu}=0$, and, therefore, the MGFs defined in (25a) and (25b) are simplified to

$$
\begin{gathered}
\varphi_{D_{I}}(s)=\frac{1}{\left|I-s C_{W} H_{I}\right|}=\prod_{k=1}^{2 L} \frac{1}{1-s \lambda_{I_{k}}} \\
\varphi_{D_{Q}}(s)=\frac{1}{\left|I-s C_{W} H_{I}\right|}=\prod_{k=1}^{2 L} \frac{1}{1-s \lambda_{Q_{k}}}
\end{gathered}
$$

where $\lambda_{I_{L}}$ and $\lambda_{Q_{L}}$ are the $l$ th eigenvalue of matrices $C_{W} H_{I}$ and $C_{W} H_{Q}$, respectively.

We assume that RVs $g_{m}$ and $y_{m}$ are i.n.d. Therefore, the eigenvalues are distinct and the characteristic functions can be partial factored as

$$
\begin{gathered}
\varphi_{D_{I}}(s)=\sum_{k=1}^{2 L} \frac{a_{I_{k}}}{1-s \lambda_{I_{k}}}, \quad a_{I_{k}}=\prod_{\substack{r=1 \\
r \neq k}}^{2 L}\left(\frac{\lambda_{I_{k}}}{\lambda_{I_{r}}-\lambda_{I_{k}}}\right) \\
\varphi_{D_{Q}}(s)=\sum_{k=1}^{2 L} \frac{a_{Q_{k}}}{1-s \lambda_{Q_{k}}}, \quad a_{Q_{k}}=\prod_{\substack{r=1 \\
r \neq k}}^{2 L}\left(\frac{\lambda_{Q_{k}}}{\lambda_{Q_{r}}-\lambda_{Q_{k}}}\right) .
\end{gathered}
$$

Using (31a) and (31b), we find closed-form expressions for the conditional probabilities in (18) and (22) as follows:

$$
\operatorname{Pr}\left(D_{I}<\left.0\right|_{x=a d+j b d}\right)=\frac{-1}{2 \pi j} \int_{\varepsilon-j \infty}^{\varepsilon+j \infty} \frac{\varphi_{D_{I}}(s)}{s} d s
$$

and

$$
\operatorname{Pr}\left(D_{Q}<\left.0\right|_{x=a d+j b d}\right)=\frac{-1}{2 \pi j} \int_{\varepsilon-j \infty}^{\varepsilon+j \infty} \frac{\varphi_{D_{Q}}(s)}{s} d s
$$

where $\varepsilon$ is a small negative number to avoid the pole at $s=0$.

Without loss of generality, we assume that $\left(\lambda_{I_{1}}, \ldots, \lambda_{I_{t}}\right)$ and $\left(\lambda_{Q_{1}}, \ldots, \lambda_{Q_{t}}\right)$ are the negative eigenvalues, and $\left(\lambda_{I_{t+1}}\right.$, $\left.\ldots, \lambda_{I_{2 L}}\right)$ and $\left(\lambda_{Q_{t+1}}, \ldots, \lambda_{Q_{2 L}}\right)$ are the positive eigenvalues. We now illustrate a common procedure for evaluating (32) and (33). Using Cauchy's residue theorem, we can write

$$
\begin{aligned}
\frac{1}{2 \pi j} \int_{C_{R}} \frac{\varphi(s)}{s} d s+ & \frac{1}{2 \pi j} \int_{\varepsilon-j \infty}^{\varepsilon+j \infty} \frac{\varphi(s)}{s} d s \\
& =\sum_{\operatorname{Re}\left(s_{i}\right)<0} \operatorname{Residue}\left(\frac{\varphi(s)}{s}, s=s_{i}\right)
\end{aligned}
$$

where $C_{r}$ is a large semicircular arc so that the resulting closed contour encloses all the singularities $s_{I}$ of $\varphi(s)$ that are located in the left-half plane (i.e., singular values whose real parts are negative). Using $s=\operatorname{Re}^{j \theta}$ and $d s=j \operatorname{Re}^{j \theta} d \theta$, we have

$$
\left|\frac{1}{2 \pi j} \int_{C_{R}} \frac{\varphi(s)}{s} d s\right| \leq\left|\frac{1}{2 \pi} \int_{C_{R}} \varphi\left(\operatorname{Re}^{j \theta}\right) d \theta\right| \leq \lim _{R \rightarrow \infty} \frac{C}{R^{2 L}} .
$$


The last term follows from the fact that $\varphi(s)$ is given by either (31a) or (31b). Equation (35) shows that as $R \rightarrow \infty$, the first integral in (34) along the semicircular arc vanishes to zero; thus, the second integral in (34) is equal to the sum of the residues. Therefore, using (31a) and (31b), and considering the negative eigenvalues only, (32) and (33) can be written as

$$
\begin{aligned}
\operatorname{Pr} & \left(D_{I}<\left.0\right|_{x=a d+j b d}\right) \\
& =-\sum_{l=1}^{t} \frac{a_{I_{l}}}{\lambda_{I_{l}}}\left(\frac{1}{2 \pi j} \int_{\varepsilon-j \infty}^{\varepsilon+j \infty} \frac{d s}{s\left(s-\frac{1}{\lambda_{I_{l}}}\right)}\right) \\
& =-\sum_{l=1}^{t} a_{I_{l}}
\end{aligned}
$$

and

$$
\begin{aligned}
\operatorname{Pr} & \left.D_{Q}<\left.0\right|_{x=a d+j b d}\right) \\
& =-\sum_{l=1}^{t} \frac{a_{Q_{l}}}{\lambda_{Q_{l}}}\left(\frac{1}{2 \pi j} \int_{\varepsilon-j \infty}^{\varepsilon+j \infty} \frac{d s}{s\left(s-\frac{1}{\lambda_{Q_{l}}}\right)}\right) \\
& =-\sum_{l=1}^{t} a_{Q_{l}}
\end{aligned}
$$

where $a_{I_{l}}$ and $a_{Q_{l}}$ are defined in (31a) and (31b).

Now that the closed-form expressions for the conditional probabilities in (13) and (17) are obtained, the BER of an arbitrary square/rectangular QAM with MRC and ICE in i.n.d. Rayleigh fading follows from (10) and (16).

2) Ricean Fading: In the case of Ricean fading in i.n.d. channels, $\bar{\mu} \neq 0$, and a closed-form expression cannot be found. Employing the result for computing the distribution of a quadratic form [14], we can compute $\operatorname{Pr}\left(D_{I}<\left.0\right|_{x=a d+j b d}\right)$ and $\operatorname{Pr}\left(D_{Q}<\left.0\right|_{x=a d+j b d}\right)$ numerically using the following equations:

$$
\begin{aligned}
& \operatorname{Pr}\left(D_{I}<\left.0\right|_{x=a d+j b d}\right)=\frac{1}{2}-\frac{1}{\pi} \int_{0}^{\infty} \frac{\sin \theta_{I}(t)}{t \rho_{I}(t)} d t \\
& \operatorname{Pr}\left(D_{Q}<\left.0\right|_{x=a d+j b d}\right)=\frac{1}{2}-\frac{1}{\pi} \int_{0}^{\infty} \frac{\sin \theta_{Q}(t)}{t \rho_{Q}(t)} d t
\end{aligned}
$$

where

$$
\begin{aligned}
\theta_{I}(t) & =\sum_{k=1}^{2 L}\left(\tan ^{-1}\left(\lambda_{a_{k}} t\right)+\frac{\left|x_{i_{k}}\right|^{2} \lambda_{a_{k}} t}{1+\lambda_{a_{k}}^{2} t^{2}}\right) \\
\rho_{I}(t) & =\prod_{k=1}^{2 L}\left(1+\lambda_{a_{k}}^{2} t^{2}\right)^{\frac{1}{2}} \exp \left(\frac{\left|x_{i_{k}}\right|^{2} \lambda_{a_{k}}^{2} t^{2}}{1+\lambda_{a_{k}}^{2} t^{2}}\right) \\
\theta_{Q}(t) & =\sum_{k=1}^{2 L}\left(\tan ^{-1}\left(\lambda_{b_{k}} t\right)+\frac{\left|x_{q_{k}}\right|^{2} \lambda_{a_{k}} t}{1+\lambda_{b_{k}}^{2} t^{2}}\right) \\
\rho_{Q}(t) & =\prod_{k=1}^{2 L}\left(1+\lambda_{b_{k}}^{2} t^{2}\right)^{\frac{1}{2}} \exp \left(\frac{\left|x_{q_{k}}\right|^{2} \lambda_{b_{k}}^{2} t^{2}}{1+\lambda_{b_{k}}^{2} t^{2}}\right)
\end{aligned}
$$

where $\lambda_{a_{r}}$ and $\lambda_{b_{r}}$ are the eigenvalues of $C_{W}^{1 / 2} H_{I} C_{W}^{1 / 2}$ and $C_{W}^{1 / 2} H_{Q} C_{W}^{1 / 2}$, respectively. As mentioned in [14], the functions

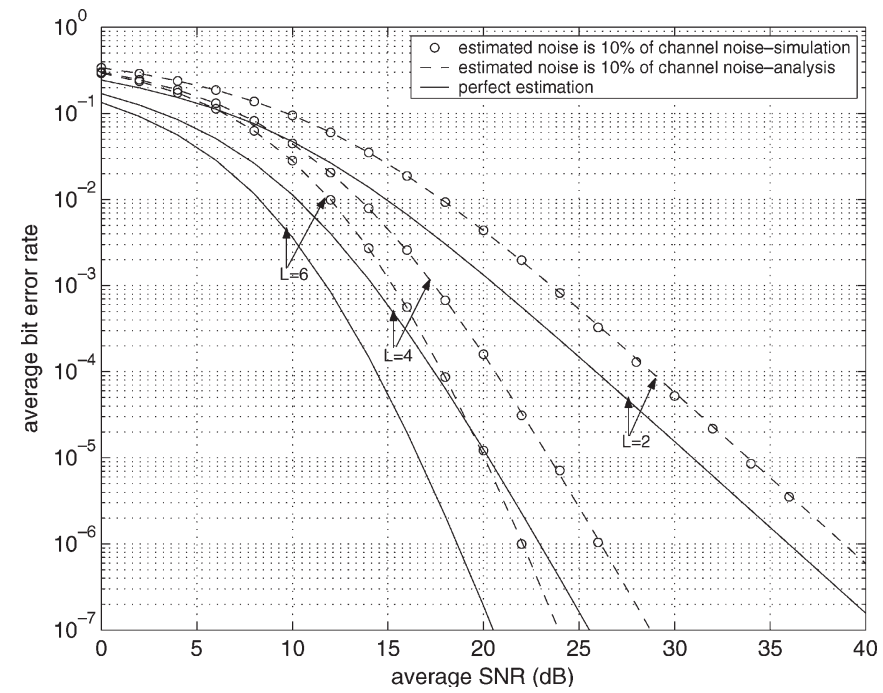

Fig. 2. Plot of BER of a 16 QAM with ICE Case 1 and $\beta=0.5$ for three different diversity branches.

$t \rho_{I}(t)$ and $t \rho_{Q}(t)$ in (38) and (39) increase monotonically toward $\infty$. Therefore, the integrals in (38) and (39) can be truncated over a finite range, with negligible error. After the computation of the conditional probabilities in (13) and (17), the BER of an arbitrary square/rectangular QAM with MRC and ICE in i.n.d. Ricean fading follows from (10) and (16).

\section{Correlated Fading}

When the diversity branches are correlated, the analysis can proceed in a similar manner to that of i.n.d Ricean fading. Note that for the correlated branches $m$ and $n$, the covariance $\sigma_{g_{m} y_{n}}$ in the covariance matrix (6) is not zero. Using the covariance and correlation matrices, the conditional probabilities in (13) and (17) can be evaluated using (38) and (39).

\section{Simulations}

All the simulations in this section are implemented in Matlab. Two ICE cases are considered as in [7]: Case 1, $\alpha=1$ and $\sigma_{S}^{2}=0.1 \sigma_{n}^{2}$, which allows for the estimation noise to be $10 \%$ of the channel noise; Case 2, in addition to the estimation noise, an irreducible error in the estimation of the channel exists, i.e., $\sigma_{S}^{2}=0.001 \sigma_{h}^{2}+0.1 \sigma_{n}^{2}$.

For i.n.d. fading channels, we consider an exponential multipath intensity profile (MIP) with the decay parameter $\beta$, i.e., $\gamma_{l}=\gamma_{1} \exp \{-\beta(l-1)\}, L=1,2, \ldots, L$.

For analytical results, we used (31) and (32) in (13) and (17) and then in (10) and (16) to obtain the BER performance of MRC QAM with ICE. In Fig. 2, the BER performance of 16 QAM with MRC for Case 1 and for three different diversity branches is presented. The MIP decay parameter $\beta$ is set to 0.5 . For both perfect and imperfect estimations, the BER performance improves when the number of diversity branches increases. Also, for Case 1, ICE does not change the slope of the BER curves. Interestingly, as the diversity order increases, the SNR difference between perfect channel estimation and ICE increases. For example, at a BER of $10^{-4}$, the SNR differences between perfect and imperfect estimations for 


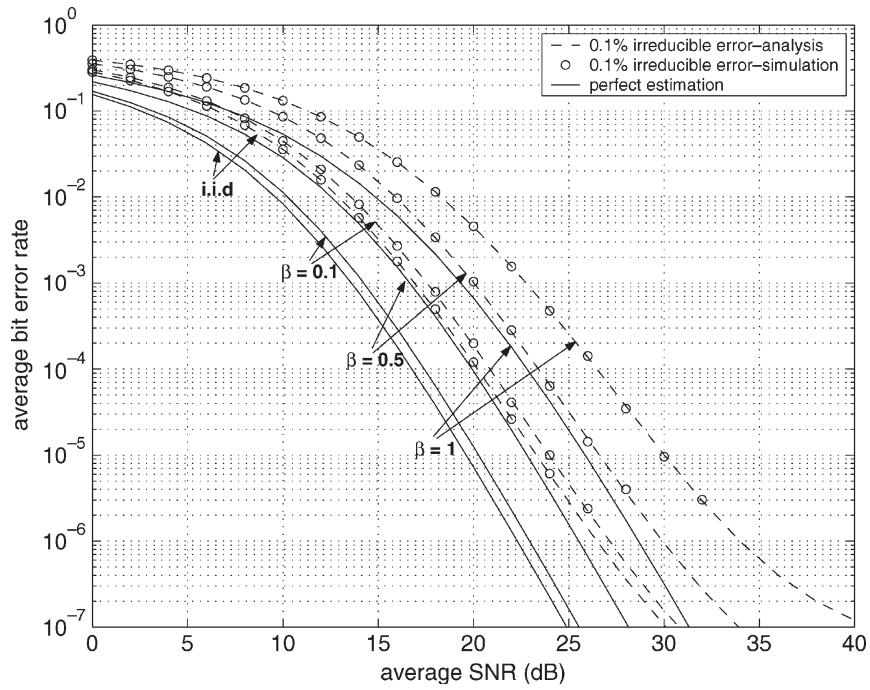

Fig. 3. Plot of BER of an 16 QAM with four diversity branches and ICE Case 2 for four different channel MIPs.

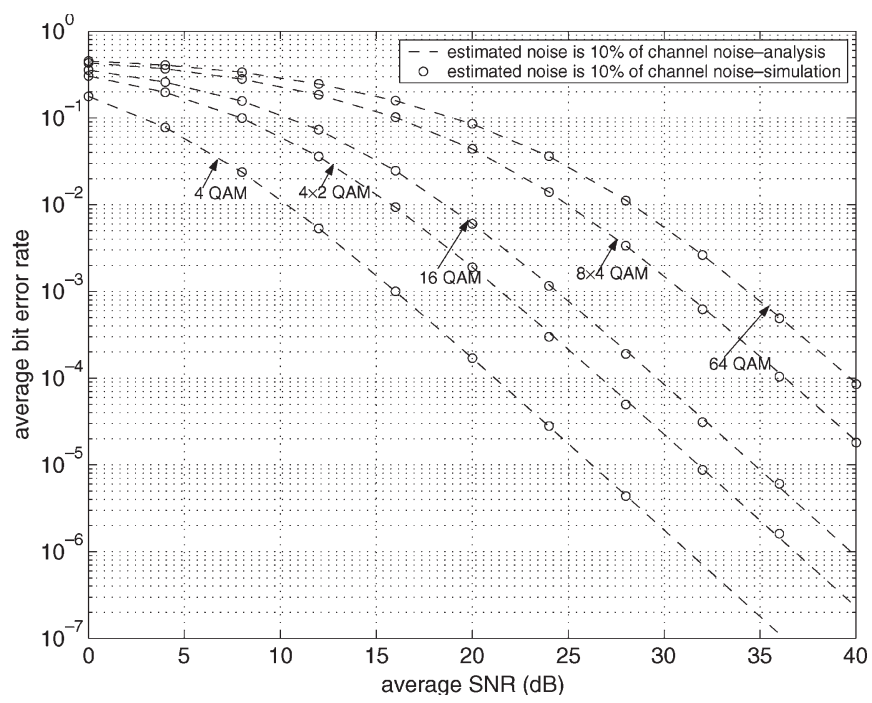

Fig. 4. Plot of BER of several QAM constellations with two diversity branches and ICE Case 1 and $\beta=0.1$.

$L=2,4$, and 6 are $2.89,3.19$, and $3.46 \mathrm{~dB}$, respectively. This shows that as the number of diversity branches increases, the ICE degradation increases rapidly.

Fig. 3 shows the BER of four branch reception of 16 QAM for four different channel MIPs for Case 2. The BER with perfect estimation for each $\beta$ is also presented for comparison. The i.i.d. channel case has the best BER performance, and it degrades as the decay exponent $\beta$ increases. For Case 2, an error floor occurs at high values of SNR.

We use (13) and (17) to plot the BER performances of several square and rectangular QAM constellations. We assume a twobranch MRC receiver, and $\beta=0.1$. The analytical results along with Monte Carlo simulations are shown in Figs. 4 and 5. For comparison between the two estimation models, we use Case 1 and Case 2 in Figs. 4 and 5, respectively, for ICE. These two figures show that to transmit an extra bit per dimension, the SNR must be increased by 3-6 dB. Also, for Case 1 (Fig. 4), both analytical and simulation results show a constant decrease

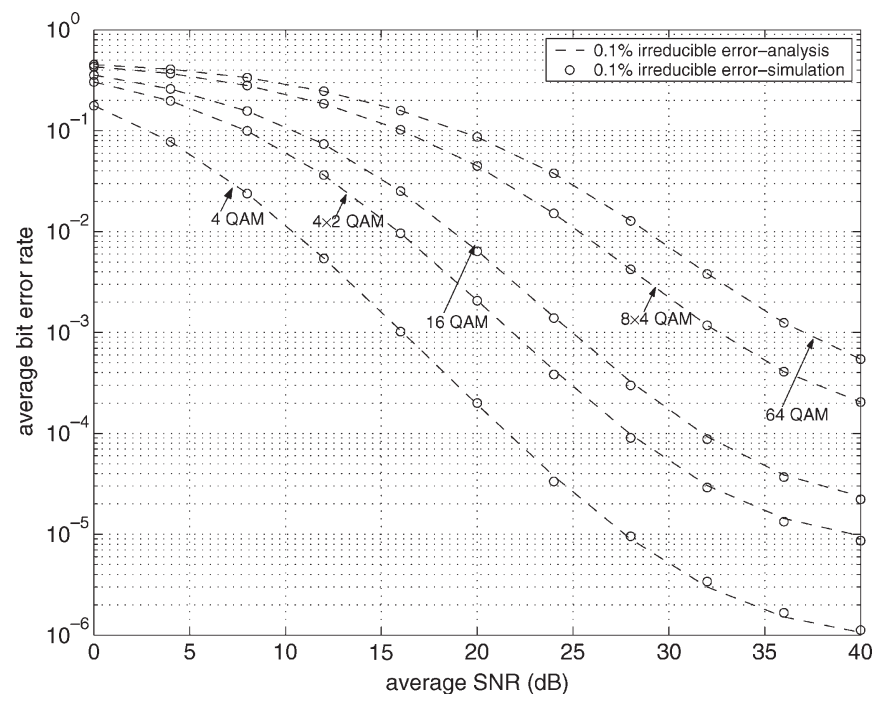

Fig. 5. Plot of BER of several QAM constellations with two diversity branches and ICE Case 2 and $\beta=0.1$.

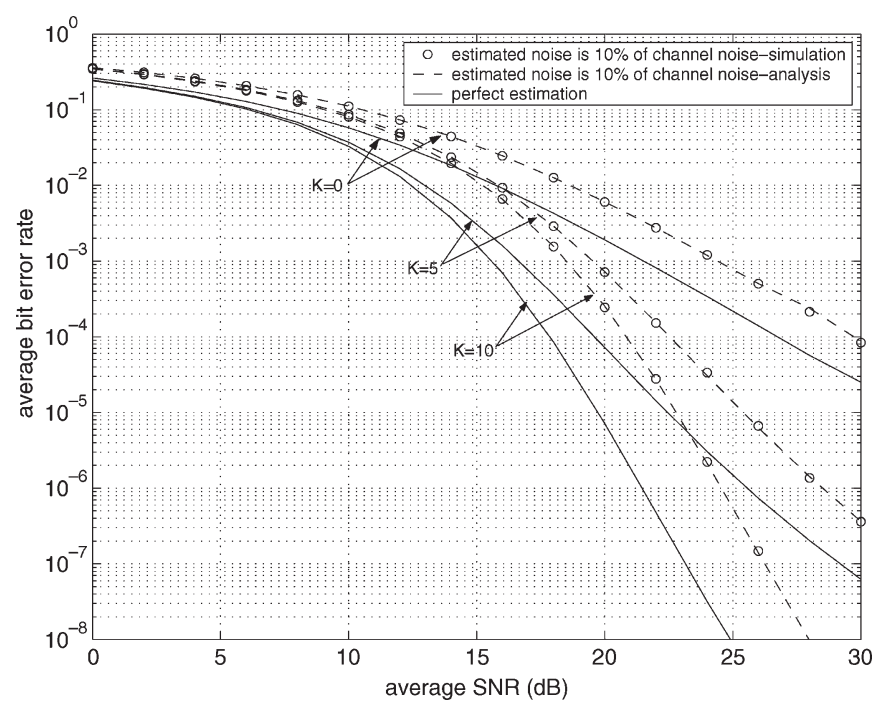

Fig. 6. Plot of BER of a 16 QAM with two diversity branches, ICE Case 1, and $\beta=0.5$ for three different Ricean factors.

while in Fig. 5 (Case 2), an error floor occurs at high values of SNR.

Fig. 6 shows the average BER of 16 QAM with two diversity branches and ICE Case 1 for different Ricean factors. Analytical bit error probability is calculated using (38) and (39) numerically. The finite range used in calculating (38) and (39) numerically needs to be increased as SNR increases. Note that the SNR difference between perfect channel estimation and ICE remains constant and does not change with the increase of the Ricean factor. As expected, the system performs better as the Ricean factor increases.

For correlated channels, we assume that the Ricean factor parameter $K$ is identical for all branches. For simplicity, we also consider equally correlated channels with the fading correlation factor $\rho$. Fig. 7 shows the BER of a two correlated branches 16 QAM with ICE Case 1 for five different correlation factors. For simulations, we have used the technique presented in [21], and (38) and (39) have been used to obtain the average BER 


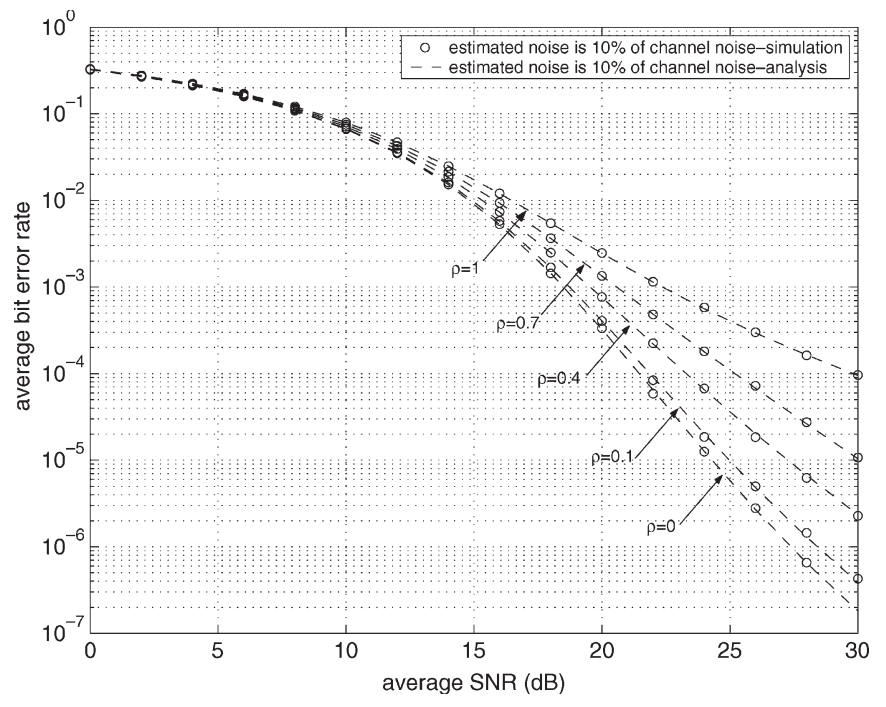

Fig. 7. Plot of BER of a 16-QAM constellations with two correlated diversity branches, ICE Case 1 , and $K=5$ for five different correlation factors.

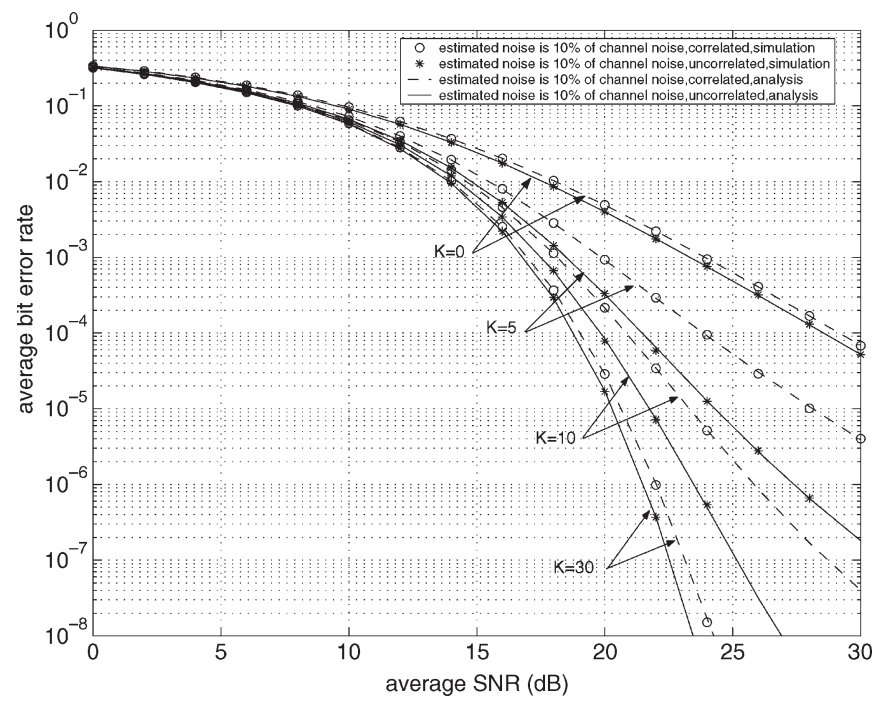

Fig. 8. Plot of BER of a 16-QAM constellations with two correlated/ uncorrelated diversity branches and ICE Case 1 for four different Ricean factors.

analytically. We observe that as $\rho$ increases, the performance of the system degrades. This is more pronounced at higher SNRs.

Fig. 8 plots the BER curves for both correlated $(\rho=0.5)$ and uncorrelated $(\rho=0)$ two diversity branches of 16 QAM with ICE Case 1, for Ricean factors $k=0,5,10$, and 30. Note that as SNR increases, the SNR difference between correlated and uncorrelated cases increases as well. Also, for $\mathrm{K}>0$ and a specific average BER, the SNR difference between the correlated and uncorrelated cases decreases as $K$ increases.

In Figs. 2-8, both simulation and analytical results are presented, and they are in excellent agreement.

\section{CONCLUSiON}

Channel estimation is an essential element of coherent digital demodulation, and ICE can severely degrade the BER of digital modulations with MRC diversity reception. The most general case involves nonidentical and possibly correlated fading, arbitrary QAM, and Ricean fading, which includes Rayleigh fading as a special case. This problem has not been comprehensively addressed before. In this paper, we analyzed the effect of ICE on the BER of an arbitrary square/rectangular Gray-coded QAM in generalized Ricean fading channels, when MRC reception is employed. We derived a general expression for the bit error probability of an arbitrary square/rectangular QAM scheme. We also derived the necessary conditional probabilities in closed form for independent and i.n.d. Rayleigh-fading channels, with MRC and ICE. An efficient numerical method has also been developed to compute the conditional probabilities for i.n.d. and correlated Ricean fading. In addition, we presented extensive Monte Carlo simulations that agreed excellently with our analytical results.

\section{REFERENCES}

[1] L. Hanzo, W. Webb, and T. Keller, Single- and Multi-carrier Quadrature Amplitude Modulation: Principles and Applications for Personal Communications, WLANs and Broadcasting. Chinchester, U.K.: Wiley, 2002.

[2] M. P. Fitz and J. P. Seymour, "On the bit error probability of QAM modulation," Int. J. Wireless Inf. Netw., vol. 1, no. 2, pp. 131-139, Apr. 1994.

[3] J. Lu, K. Letaief, J. C.-I. Chung, and M. L. Liou, "M-PSK and M-QAM BER computation using signal-space concepts," IEEE Trans. Commun., vol. 47, no. 2, pp. 181-184, Feb. 1999.

[4] L. Yang and L. Hanzo, "A recursive algorithm for the error probability evaluation of M-QAM," IEEE Commun. Lett., vol. 4, no. 10, pp. 304306, Oct. 2000.

[5] K. Cho and D. Yoon, "On the general BER expression of one- and two dimensional amplitude modulations," IEEE Trans. Commun., vol. 50, no. 7, pp. 1074-1080, Jul. 2002.

[6] X. Tang, M.-S. Alouini, and A. J. Goldsmith, "Effect of channel estimation error on M-QAM BER performance in Rayleigh fading," IEEE Trans. Commun., vol. 47, no. 12, pp. 1856-1864, Dec. 1999.

[7] S. K. Wilson and J. M. Cioffi, "Probability density functions for analyzing multi-amplitude constellations in Rayleigh and Ricean channels," IEEE Trans. Commun., vol. 46, no. 3, pp. 380-386, Mar. 1999.

[8] L. Cao and N. C. Beaulieu, "Exact error rate analysis of diversity 16-QAM with channel estimation error," IEEE Trans. Commun., vol. 52, no. 6, pp. 1019-1029, Jun. 2004.

[9] - "Closed-form BER results for MRC diversity with channel estimation errors in Ricean fading channels," IEEE Trans. Wireless Commun., vol. 4, no. 4, pp. 1440-1447, Jul. 2005.

[10] Y. Ma, R. Schober, and S. Pasupathy, "Effect of imperfect channel estimation on MRC diversity in fading channels," in Proc. IEEE Int. Conf. Commun., Jun. 2004, pp. 3163-3167.

[11] _ - "Performance of M-PSK with GSC and EGC with Gaussian weighting errors," IEEE Trans. Veh. Technol., vol. 54, no. 1, pp. 149-162, Jan. 2005.

[12] L. Najafizadeh and C. Tellambura, "Exact BER analysis of an arbitrary square/rectangular QAM for MRC diversity with ICE in nonidentical Rayleigh fading channels," in Proc. IEEE Int. Conf. Commun., 2005, vol. 4, pp. 2387-2391.

[13] J. G. Proakis, Digital Communications, 4th ed. New York: McGrawHill, 2001.

[14] J. P. Imhof, "Computing the distribution of quadratic form in normal variables," Biometrika, vol. 48, no. 3/4, pp. 419-426, Dec. 1961.

[15] J. K. Cavers, "An analysis of pilot-symbol-assisted modulation for Rayleigh fading channels," IEEE Trans. Veh. Technol., vol. 40, no. 4, pp. 1389-1399, Nov. 1991

[16] Y.-S. Kim, C.-J. Kim, G.-Y. Jeong, Y.-J. Bang, H.-K. Park, and S. S. Choi, "New Rayleigh fading channel estimator based on PSAM channel sounding technique," in Proc. IEEE Int. Conf. Commun., Jun. 1997, vol. 3, pp. $1518-1520$.

[17] M. M. Wang, W. Xiao, and T. Brown, "Soft decision metric generation for QAM with channel estimation error," IEEE Trans. Commun., vol. 50, no. 7, pp. 1058-1061, Jul. 2002.

[18] W. Xiao, "Optimal detection of M-QAM signal with channel estimation error," in Proc. IEEE Int. Conf. Commun., May 2003, vol. 5, pp. 3251-3255. 
[19] M. Schwartz, W. Bennett, and S. Stein, Communication Systems and Techniques. New York: McGraw-Hill, 1966.

[20] A. M. Mathai and S. Provost, Quadratic Forms in Random Variables: Theory and Applications. New York: Marcel Dekker, 1992.

[21] Y. Chen, "New diversity combiners and the impact of fading correlation on diversity systems," M.S. thesis, Dept. Elect. Comput. Eng., Univ. Alberta, Edmonton, AB, Canada, 2004.

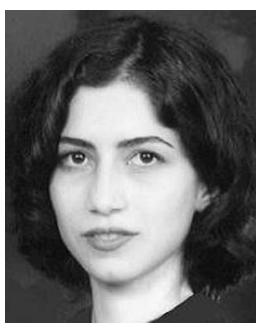

Laleh Najafizadeh (S'02) received the B.Sc. degree from Isfahan University of Technology, Isfahan, Iran, in 1999 and the M.Sc. degree from the University of Alberta, Edmonton, AB, Canada, in 2003, both in electrical engineering. Currently, she is working toward the Ph.D. degree at Georgia Institute of Technology, Atlanta.

From 2003 to 2004, she was with iCORE Wireless Communications Laboratory at the University of Alberta. Her research interests are in the areas of fading channels, channel estimation, and CMOS and SiGe analog circuits.

Ms. Najafizadeh was the recipient of a Delta Kappa Gamma World Fellowship award and postgraduate scholarships from the Alberta Ingenuity Fund and the Alberta Informatics Circle of Research Excellence.

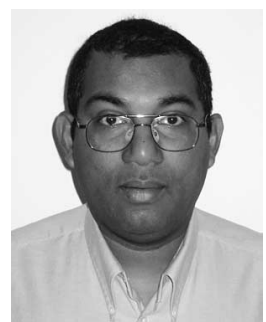

Chintha Tellambura (SM'02) received the B.Sc. degree (with first-class honors) in telecommunications and electronics from the University of Moratuwa, Moratuwa, Sri Lanka, in 1986, the M.Sc. degree in electronics from the University of London, London, U.K., in 1988, and the Ph.D. degree in electrical engineering from the University of Victoria, Victoria, BC, Canada, in 1993.

He was a Postdoctoral Research Fellow with the University of Victoria from 1993 to 1994, and with the University of Bradford, Bradford, U.K., from 1995 to 1996 . He was with Monash University, Melbourne, Australia, from 1997 to 2002. Currently, he is an Associate Professor with the Department of Electrical and Computer Engineering, University of Alberta, Edmonton, $\mathrm{AB}$, Canada. His research interests include coding, communication theory, modulation, equalization, and wireless communications.

Prof. Tellambura is an Associate Editor for both the IEEE TRANSACTIONS on Communications and the IEEE TRANSACTIONS ON Wireless Communications. He is a Cochair of the Communication Theory Symposium in Globecom'05, which was held in St. Louis, MO. 